\title{
Soft Computing Techniques For Process CONTROL APPLICATIONS
}

\author{
Rahul Malhotra ${ }^{1}$, Narinder Singh ${ }^{2}$, Yaduvir $\operatorname{Singh}^{3}$ \\ ${ }^{1}$ Research Scholar, Punjab Technical University, Punjab, India \\ ${ }^{1}$ blessurahul egmail . com \\ ${ }^{2,3}$ Thapar University, Patiala, Punjab, India \\ ${ }^{3}$ yad_praeyahoo.com
}

\begin{abstract}
Technological innovations in soft computing techniques have brought automation capabilities to new levels of applications. Process control is an important application of any industry for controlling the complex system parameters, which can greatly benefit from such advancements. Conventional control theory is based on mathematical models that describe the dynamic behaviour of process control systems. Due to lack in comprehensibility, conventional controllers are often inferior to the intelligent controllers. Soft computing techniques provide an ability to make decisions and learning from the reliable data or expert's experience. Moreover, soft computing techniques can cope up with a variety of environmental and stability related uncertainties. This paper explores the different areas of soft computing techniques viz. Fuzzy logic, genetic algorithms and hybridization of two and abridged the results of different process control case studies. It is inferred from the results that the soft computing controllers provide better control on errors than conventional controllers. Further, hybrid fuzzy genetic algorithm controllers have successfully optimized the errors than standalone soft computing and conventional techniques.
\end{abstract}

\section{KEYWORDS}

EVOLUTIONARY ALGORITHMS, FUZZY LOGIC, GENETIC ALGORITHMS, DTC INDUCTION MOTOR, TURBINE COMPRESSOR SYSTEM, DC SERVO MOTOR

\section{INTRODUCTION}

Exponential growth in soft computing technologies has marked new milestones in powerful representation, modelling paradigms and optimization mechanisms for solving modern controller issues. Soft computing has provided sophisticated methodology for the development of industrial process controllers. It is considered to be a state-of-art approach to artificial intelligence. With the emergence of high performance computing power, design engineers have applied artificial intelligence techniques to a wide spectrum of real-world problems in intelligent and autonomous control. Within the last decades, substantial amount of growth has been noticed on the application of soft computing techniques in engineering. The pervasive use of this technique in various engineering applications makes it an indispensible tool. The principal constituents of soft computing include theory of neurons, fuzzy logic, evolutionary computing, genetic algorithms, chaostic systems and probabilistic reasoning. Out of which the two emerging techniques viz. fuzzy logic and genetic algorithms are considered in this research work to control the process of the systems. 
Soft computing techniques have been recognized as attractive alternatives to the standard, well established hard computing paradigms. Soft computing is still in its initial stages of crystallization. Soft computing techniques, in comparison with hard computing employ different methods which are capable of representing imprecise, uncertain and vague concepts. Soft computing techniques are able to handle non-linearity and offers computational simplicity.

Fuzzy logic is a universal approximator of any multivariate function because it can be used for modelling highly non-linear, unknown or partially known controllers, plants or processes. Fuzzy logic helps an engineer for solving non-linear control problems in process control applications. It emulates human reasoning and provides an intuitive way to design functional block for an intelligent control system.

Genetic algorithms (GAs) have emerged as potentially robust optimization tools in the last decades. Genetic algorithms (GAs) are a search heuristic that mimics the process of natural evolution. Genetic algorithms (GAs) can be applied to the process controllers for their optimization using natural operators viz. mutation and crossover [18]. Well established methodologies have been discussed in literature for integrating soft computing techniques to realize synergistic or hybrid models with which better results could be obtained.

Simulation is the computational realization of a model. They are executable, live representation of models that can be as meaningful as the real experiments. Simulation allows an engineer to reason if a model makes sense or not and how the model behaves for the certain parameter variations. Simulations can be carried out for designing and implementation of conventional proportional integral derivative (PID) controllers, fuzzy logic controllers (FLC) and hybrid fuzzy logic genetic algorithms (HFLGA) controllers. Simulation applications can dynamically adjust the various process control parameters at running state of the plant.

\section{FUZZY LOGIC}

Fuzzy logic attempts to systematically and mathematically emulate human reasoning and decision making [10]. It provides an intuitive way to implement control systems, decision making and diagnostic systems in various branches of industry. Fuzzy logic represents an excellent concept to close the gap between human reasoning and computational logic. Variables like intelligence, credibility, trustworthiness and reputation employ subjectivity as well as uncertainty. They cannot be represented as crisp values, however their estimation is highly desirable. Fuzzy systems are emerging technologies targeting industrial applications and added a promising new dimension to the existing domain of conventional control systems. Fuzzy logic allows engineers to exploit their empirical knowledge and heuristics represented in the IF-THEN rules and transfer it to a functional block. Fuzzy logic systems can be used for advanced engineering applications such as intelligent control systems, process diagnostics, fault detection, decision making and expert systems.

\section{Genetic Algorithms (GAs)}

Evolutionary algorithms (EAs) are population-based meta heuristic optimization algorithms that use biology-inspired mechanisms and survival of the fittest theory in order to refine a set of solution iteratively [7]. Genetic algorithms (GAs) are subclass of evolutionary algorithms (EAs) where the elements of the search space are binary strings or arrays of other elementary types. Genetic algorithms (GAs) are computer based search techniques patterned after the genetic mechanisms of biological organisms that have adapted and flourished in changing highly competitive environment. Last decade has witnessed many exciting advances in the use of genetic algorithms (GAs) to solve optimization problems in process control systems. Genetic algorithms (GAs) are the solution for optimization of hard problems quickly, reliably and accurately. As the 
complexity of the real-time controller increases, the genetic algorithms (GAs) applications have grown in more than equal measure.

\section{Conventional Controllers}

The important issue in the modern control industries is to develop methodologies, concepts, algorithms, technologies for the design of process control systems which must be able to evolve, self develop, self organize, self evaluate and to self improve $[6,22]$. Over the years, control of processes and systems in the industry is customarily done by experts through conventional proportional integral derivative (PID) controllers because of its simplicity, low cost design and robust performance in wide operating conditions. Conventional control systems suffer from transient and steady state problems like overshoot, settling time and rise time. Various technologies and modifications have been employed to overcome these difficulties, which includes auto tuning of proportional integral derivative (PID) controllers, adaptive techniques and compensation techniques. Automatic tuning procedures are required for satisfactory control of controller parameters. The concept of evolving intelligent techniques was established as a synergy between conventional systems, fuzzy systems and genetic algorithms as the structures for representation of information and real time methods for machine learning. Soft computing methodologies have shown to be well suited to deal with significant uncertainties that may encounter in solving real world problems. Hybridization of the controller structures comes to one's mind immediately to exploit the beneficial sides of the two categories of soft computing techniques. This thesis work is intended to design a process control system using conventional proportional integral derivative (PID) controllers so that a further improved performance in both the transient and steady state can be achieved, when compared to the system response obtained when either classical proportional integral derivative (PID), fuzzy logic controller (FLC) or hybridized fuzzy logic genetic algorithm (HFLGA) controller is implemented.

\section{Fuzzy Logic ConTrollers}

Analytical studies on transient response, stability and reliability gives dynamical performance of conventional proportional integral derivative (PID) controllers in normal operating conditions. However, the capacity of conventional controllers is significantly reduced when applied to systems with non-linearities. Fuzzy systems can improve the performance of conventional proportional integral derivative (PID) controllers. A number of approaches have been proposed and implemented fuzzy control systems for controlling the process. The aim of incorporating fuzzy techniques in process control systems is to get ahead of the limits of conventional techniques and to improve the existing tools by optimizing the dynamical performances. Fuzzy control is a versatile and effective approach to deal with non-linear and uncertain system. A fuzzy control process can be performed by fuzzification, fuzzy inference and defuzzification components as shown in figure 1.

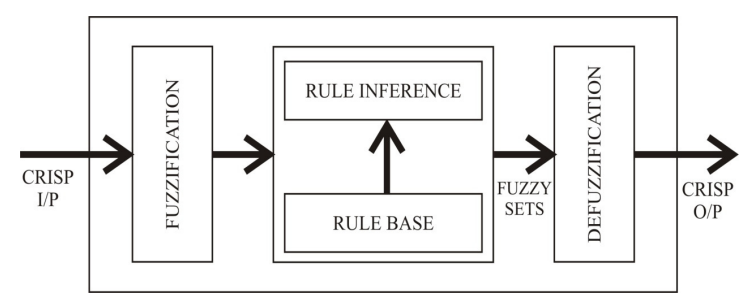

Figure 1: Schematic of fuzzy logic control. 
A typical fuzzy control system as applied to a plant comprises of sensory data which is applied for analog to digital conversion followed by crisp to fuzzy conversion which is further given to an inference engine. On the other hand, fuzzy to crisp conversion followed by digital to analog conversion takes place, which is applied as an input to control the plant with fuzzy means. The process is shown in figure 2 .

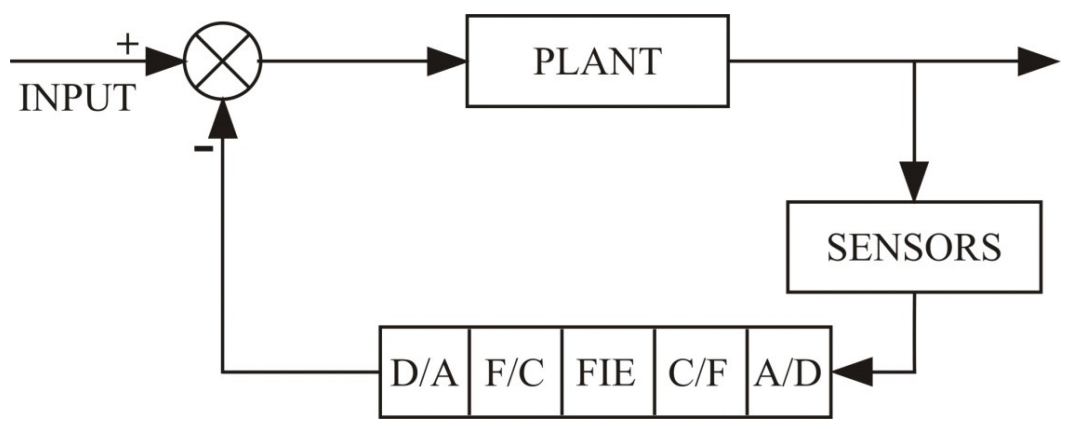

D/A: Digital to Analog conversion

A/D: Analog to digital conversion

$\mathrm{C} / \mathrm{F}$ : Crisp to fuzzy

F/C: Fuzzy to Crisp

FIE: Fuzzy Inference Engine

Figure 2: Systematic fuzzy process control system

The cornerstone of any fuzzy controller is its inference engine, which consists of set of rules that reflect knowledge base and reasoning structure for the solution of any problem. Fuzzy control methods are critical for meeting the demands of complex non-linear systems as they bestow robust, adaptive, self character to complex systems that demand high stability and functionality beyond the capabilities of traditional methods. Fuzzy systems and fuzzy control theories have added a new dimension to control systems engineering. The more recent and rigorous approaches to fuzzy control theory, make it an integral part of modern control theory. A fuzzy logic controller can produce arbitrary non-linear control law and the lack of systematic procedure for the configuration of its parameters remains the main obstacle in practical applications. The design of fuzzy logic controllers has been controlled with genetic algorithms to further optimize the controller parameters.

\section{GENETIC AlgORITHMS (GAS) CONTROLlERS}

Analytical Genetic algorithms (GAs) are deployed for optimal selection of antecedents and consequents in fuzzy systems. Genetic algorithms (GAs) have been proven to be powerful in optimization, design and real time implementation. Genetic algorithms (GAs) which are modelled on natural evolutionary strategies are a methodology that has been introduced as a leaning and optimization technique for solving complex problems. Furthermore, genetic algorithm (GAs) search has inherent parallelism which enables rapid identification of high performance regions of complex domains without experiencing problems with high dimensionality. Thus, genetic algorithms (GAs) have found exponential growth in many control applications especially while integrating the fuzzy logic, where they have applied to the process of learning control rules, selection of rules and their membership functions. The theory of genetic algorithms (GAs) is based upon initialization of chromosomes, giving fitness value to the chromosome according to their performance criteria, reproduction based on probability, crossover which divides the binary coding of each parent into two or more segments and then combines to give a new offspring that 
has inherited part of its coding from each parent, mutation process in which the coding of offsprings is done with low probability.

These optimization algorithms perform a stochastic search by iterations of populations of solutions according to their fitness. In control applications, fitness is related to performance measures of the process controllers. Performance of fuzzy logic controllers can be improved if fuzzy reasoning model is supplemented by genetic algorithm mechanism. The genetic algorithm enables us to generate an optimal set of parameters for the fuzzy logic model.

\section{HybridizATION OF SOFT COMPUTING TEChNIQUES}

Hybridization of intelligent systems is a promising field of modern intelligence for the development of next generation controllers. Integration of soft computing techniques can solve tough problems in process control industries. Hybrid artificial intelligence techniques provide more robust and reliable problem solving models than standalone models. Integrating these techniques enhance the overall strengths and lessen weakness thereby helping to solve overall control problem in effective way. Various strategies, models and design have been suggested by researchers to integrate various intelligent systems for practical applications. Some of them are based on functionality and characteristics of intelligent systems while others are based on techniques and mechanisms used to integrate the systems. The ultimate goal of integration is to model the problem by taking advantages of strengths to achieve effectiveness and efficiency. Mathematical models can be used in conjunction with intelligent techniques to improve the performance of hybrid systems for real world applications. One of the main problems of hybridization is to make a prudent combination of artificial intelligence methodologies to built hybrid intelligent models that can outperform the results of an individual intelligent method. New applications to real world problems of hybrid integrated systems are found to achieve better results than standalone techniques. After many years of efforts towards augmenting fuzzy systems with learning and adaptation capabilities, evolutionary computing has resulted in the emergence of fuzzy genetic hybrid system with the adaptation capabilities of evolutionary algorithms. Fuzzy systems have demonstrated the ability to formalize the approximate reasoning typical of humans in computational efficient manner. Genetic algorithms, on the other hand, constitute a robust technique in complex optimization, identification, learning and adaptation problems. Their confluence leads to increase capabilities for the design and optimization of fuzzy systems. The primary goal of artificial intelligence is to produce intelligent machines which simulate and emulate human beings intelligence.

The parameters of fuzzy system are specified by human designer. Following their successful application to a variety of learning and optimization problems, genetic algorithms have been proposed as a learning method that can enable automatic generation of optimal parameters for fuzzy controllers, based on some objective criteria. Genetic algorithm can be applied with fuzzy in three ways. In the first case, linguistic rules of fuzzy controller are fixed and their membership functions are to be optimized. In the second case the membership functions of linguistic values are fixed and the optimal sets of rules for the problem are determined by genetic algorithms. In the third approach, the rules and membership functions are adjusted simultaneously.

Ever since fuzzy logic was introduced by Professor Lotfi A. Zadeh in mid sixties and genetic algorithms (GAs) Professor John Holland in early seventies, these two fields are widely been a grey area of research around the globe. During the last few years, they have been experiencing extremely rapid growth in industrial world, and considered as very effective tools for solving realworld problems. The genetic algorithms (GAs) are an efficient and robust approach for generating fuzzy rules. The integration of fuzzy logic theory with genetic algorithms (GAs) have two functions. Firstly genetic algorithms (GAs) are used to optimize the parameters of fuzzy logic and 
secondly fuzzy logic automatically modifies genetic parameters such as mutation, crossover rate during the optimization process. The algorithm for performing the fuzzy logic genetic algorithm regression can be summarized as follows:

Step I Mapping solution space into search space, that is, binary strings. Construct fuzzy fitness function using an objective function.

Step II Create initial random population, that is, a population of fuzzy regression coefficient which is randomly specified.

Step III Evaluate each chromosome in the population in terms of its fitness value.

Step IV If termination conditions exist, go to step VIII.

Step V Generate new population using selection methods, which randomly selects the chromosome from the current population.

Step VI Create new chromosomes by mating randomly selected chromosomes. The resulting offspring replaces the original parent chromosome in the population.

Step VII Mutate some randomly selected chromosomes with their specified possibility.

Step VIII Stop, return the best chromosome and translate it into fuzzy coeffiecient.

The stop criteria may be maximum or minimum number of generations. This interactive process can be used to obtain the improved performance of fuzzy coefficient. The block diagram for hybrid fuzzy logic genetic algorithm (HFLGA) approach on the basis of the above algorithm is shown in Figure 3.

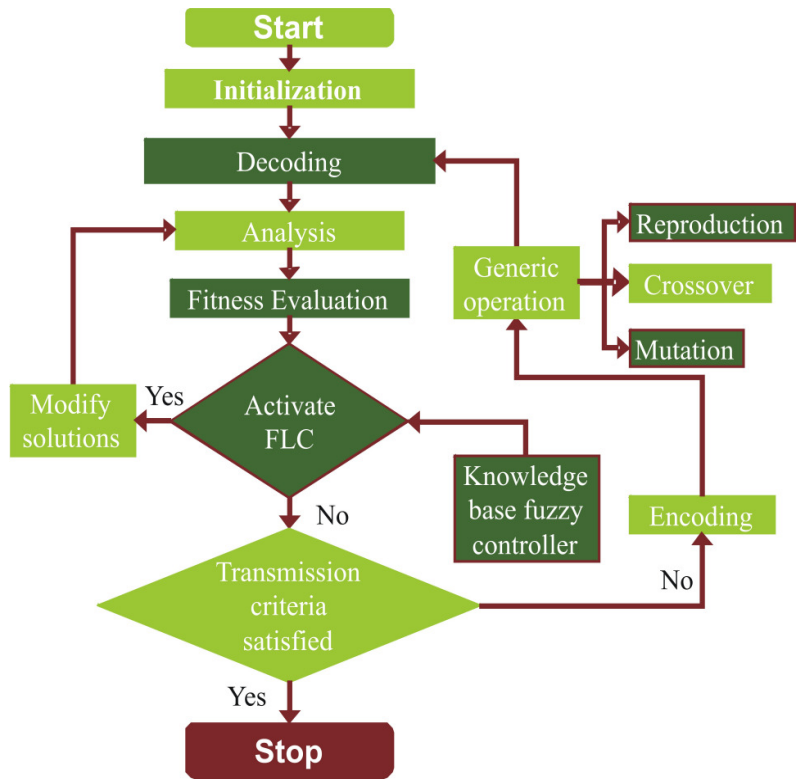

Figure 3: Block diagram of hybrid fuzzy logic genetic algorithm

Initial population is to be produced by selection and encoding techniques and the computation is performed with genetic operators to evaluate the fitness function with respect to an objective function. As a result, computing time and the risk of premature convergence is remarkably reduced. Hybridization of fuzzy logic with genetic algorithms can better optimize the controller parameters. 


\section{Case Study I - Direct Torque Control of Induction Motor}

First of all, artificial intelligence techniques are incorporated to tackle the inherent disadvantages of conventional direct torque control schemes, such as starting problems from null states, the requirement of torque and flux estimators $[1,2]$. In a conventional direct torque control method, voltage is applied for the entire period, which in turn produces high stator current and electromagnetic torque with a result high torque ripples are produced during motor operations [3].

An intelligent technique to analyze high performance decoupled flux and torque control have been implemented in this work. Fuzzy logic is used to select an inverter state to achieve the torque and flux reference values. For experimentation, a 3-phase, 1-kilowatt, $1300 \mathrm{rpm}$ induction motor drive is taken up. Triangular membership functions are used to represent the flux errors, electromagnetic torque errors and flux angle. Fuzzy rules are generated by trial and error method for the selection of proper inverter state. A motor is subjected to a frequency of $10 \mathrm{KHz}$, sampling time of $0.1 \mathrm{~ms}$, and having torque and flux reference values of $2.5 \mathrm{Nm}$ and $0.5 \mathrm{~Wb}$ respectively. Genetic algorithms have been applied for 150 generations having population size of 20 chromosomes. $50 \%$ of crossover rate and $10 \%$ of mutation rate with a single point crossover is used during simulations. Roulette wheel selection method with normalized fitness values is used for selection of chromosomes. The simulation studies show a maximum improvement of $23 \%$ in flux error for fuzzy logic and hybrid fuzzy logic genetic algorithm controller. For operating conditions $a=100 \%, b=100 \%$, flux error of $2.5 \mathrm{e}-3$ and 1.8 e-3 have been noted for fuzzy logic controller and hybrid fuzzy logic genetic algorithm controller respectively. Simulation studies are carried out on different operating conditions of the motor. It is inferred from the simulation results that the improvement of flux error in fuzzy logic standalone and hybrid fuzzy logic genetic algorithm controllers is $23 \%, 22 \%, 19 \%, 18 \%$ and $17 \%$ for operating conditions a $=100 \%$, $b=100 \%$. Further, for operating conditions $a=50 \%, b=50 \%$, performance improvement in flux errors ranges from $10 \%$ to $2 \%$ for fuzzy logic standalone and hybrid fuzzy logic genetic algorithm controllers respectively. For the above operating conditions, the percentage improvement in torque error has also been noted. For the operating condition $a=100 \%, b=100 \%$, the performance improvement of about 3\% is noted in fuzzy logic standalone and hybrid fuzzy logic genetic algorithm controllers. For different operating conditions the performance improvement is noted. It is inferred from the above simulation results that the performance improvement in flux error and torque error is more at initial speed of motor. In nutshell, fuzzy logic controller based on genetic algorithm technique outperforms the fuzzy logic standalone controllers.

\section{Case Study II - Gas Turbine Speed Control System}

Gas turbines are highly non linear plants that have multiple inputs and multiple outputs [4]. Due to high rotational and high temperature of gas turbines, operational parameters must be closely maintained and tuned. Turbine speed control system is taken up to be controlled with artificial intelligent techniques because it is often encountered in refineries in the form of steam turbine that uses hydraulic governor to control the speed of turbine [33]. A conventional proportional integral derivative (PID) controller can be used to control the turbine compressor system. It is a feedback control system which measures the output variable and sends the control signal to the controller. The controller compares the value of the output signal with the reference value and gives the control signal to the final control element via the actuator. The Ziegler-Nichols (Z-N) methods rely on open-loop step response or closed-loop frequency response tests. A proportional integral derivative (PID) controller is tuned according to a table based on the process response test. According to Zeigler-Nichols frequency response tuning criteria $K_{p}=0.6 K_{c u}, \quad \tau_{i}=0.5 T$ and $\tau_{d}=0.125 T$. 
For the proportional integral derivative (PID) controller in this case study, the values of tuning parameters obtained are $\mathrm{K}_{\mathrm{p}=18}, \tau_{\mathrm{i}}=1.4, \tau_{\mathrm{d}}=0.3$ and $\mathrm{P}=30, \mathrm{I}=21.4, \mathrm{D}=9$.

Usually, initial design values of proportional integral derivative (PID) controller obtained by all means needs to be adjusted repeatedly through computer simulations until the closed loop system performs or compromises as desired. The computations were carried out in Simulink.

The proportional integral derivative (PID) controller gives an overshoot of $52.4 \%$, settling time and peak time of 9.66 seconds and 10.9 seconds respectively, which is quite high and will produce high distortions in the system.

Due to high transient response in proportional integral derivative (PID) controller, output is greatly distorted and the efficiency of the plant is considerably reduced [6]. The fuzzy proportional integral derivative (PID) controllers are the natural extension of their conventional version, which preserve their linear structure of proportional integral derivative (PID) controller. The fuzzy proportional integral derivative (PID) controllers are designed using fuzzy logic control principle in order to obtain a new controller that possesses analytical formulas very similar to digital proportional integral derivative (PID) controllers. In this experimental study, classic interpretation of Mamdani logic operations is applied. The fuzzy logic controller (FLC) gives much lower overshoot, settling time and peak time than the conventional proportional integral derivative (PID) controller. Typical value of overshoot is $8.75 \%$ and settling time and peak time are 6.8 seconds and 6.2 seconds respectively, which shows the comparative improvement in the fuzzy logic based controller than conventional proportional integral derivative (PID) controller for different process control parameters.

Genetic algorithms (GAs) are intelligent optimization technique [18] that relies on the parallelism found in nature, in particular its searching procedures which are based on the mechanics of natural selection and genetics. Genetic algorithms, with a population size of 20 chromosomes, run for 50 generations. Roulette wheel method is used for selection of chromosomes, with a two point crossover, having mutation probability 0.01 .

The step response of hybrid fuzzy genetic algorithms (HFLGA) system shows that the transient response parameters are better optimized than fuzzy logic controller (FLC) and conventional proportional integral derivative (PID) controller. This shows the superiority of hybrid fuzzy genetic algorithms (HFLGA) over conventional controllers and standalone fuzzy controllers.

To verify the results of Simulink based model of turbine compressor system using optimum block set, m-file were also generated using MATLAB commands. Through comparative analysis of flow control of turbine compressor system, hybrid fuzzy genetic algorithms (HFLGA) shows almost negligible overshoot of about $1 \%$ and the remarkable improvement in settling time and peak time whose typical values are about 3.7 seconds and 4.1 seconds respectively.

The comparison of the error performance parameters integral of absolute error (IAE) and integral of time and absolute error (ITAE) of proportional integral derivative (PID) controller, fuzzy logic controller (FLC) and hybrid fuzzy genetic algorithm (HLFGA) controller shows the superiority of hybrid controllers over stand alone and conventional proportional integral derivative (PID) controllers. The integral of absolute error (IAE) and integral of time and absolute error (ITAE) of proportional integral derivative (PID) controller is 0.98 and 1.94 respectively. The integral of absolute error (IAE) and integral of time and absolute error (ITAE) of fuzzy logic controller (FLC) are 0.83 and 0.91 respectively. The integral of absolute error (IAE) and integral of time and absolute error (ITAE) of hybrid fuzzy genetic algorithm (HLFGA) controller is 0.76 and 0.98 , which is remarkably less and shows the optimized control of the hybrid controllers on the errors of the gas turbine. 


\section{CaSe Study III - Intelligent Control OF DC Servo Motor}

Servo mechanisms are receiving wide attention for industrial applications because of their high torque density, high efficiency and small size [13]. They are also known as control motors. Due to multivariate and non-linear in nature, it is difficult to achieve the optimum speed of the motor. Conventional proportional integral (PI) controllers suffer from their inherent losses like maximum overshoot, high settling time and peak time. The fuzzy control has been focus to control the speed of servo motor. The auto tuning for fuzzy logic controller based on genetic algorithm fine tune the controller parameters. In this case study, proportional integral (PI) controllers, fuzzy logic controllers and genetic algorithm base controllers are applied to $1200 \mathrm{rpm}, 120 \mathrm{~W}, 240 \mathrm{~V} \mathrm{DC}$ servo motors. For the armature voltage control, simulations are performed at different armature voltages, and the voltage applied to field circuit is kept constant. After simulation, the various control parameters of speed control of DC servo mechanism using proportional integral (PI) were investigated. A maximum overshoot of 45.33 percent and a settling time and peak time of 2.5 seconds and 3.1 seconds respectively are noted. Due to high overshoot, settling time and peak time of the plant parameters and the non-linear operating conditions, proportional integral (PI) controllers may become unable to provide the required control performance. These inherent disadvantages of proportional integral (PI) controller have encouraged the replacement of the conventional proportional integral (PI) controller with soft computing techniques such as fuzzy logic controller (FLC) and hybrid fuzzy logic genetic algorithm (HFLGA) controllers.

A maximum overshoot of 9.33 percent, settling time and peak time of 1.8 seconds and 2.3 seconds are noted which are comparably less than conventional proportional integral (PI) controller. Fuzzy logic controllers can cope with non-linearity, load disturbances but tuning of fuzzy logic (FL) parameters and stabilities are its main problems. Genetic algorithms are useful approach to the problems requiring effective and efficient searching. This strategy is proposed to optimize the performance of the fuzzy logic controlled speed control of DC motor. Genetic algorithms with a population size of 20 chromosomes run for 50 generations. Roulette wheel method is used for selection of chromosomes, with a two point crossover, having mutation probability 0.01 . In this case, the fitness function to evaluate the individuals of each generation can be chosen to be the reciprocal of integral time of absolute error (ITAE).

After simulation, it is investigated that the overshoot becomes 3.2 percent and settling time and peak time are noticed about 1.5 seconds and 1.7 seconds respectively which shows a significant improvement than fuzzy logic (FL) standalone controller and the conventional proportional integral (PI) controller. During the search process, the genetic algorithm looks for the optimum setting of proportional integral (PI) speed controller gains which minimizes the performance indices viz. integral of absolute error (IAE) and integral of time and absolute error (ITAE). The lowest value of 0.08 and 0.001 have been noted for integral of absolute error (IAE) and integral of time and absolute error (ITAE) when simulated in fuzzy logic based genetic algorithm controllers, whereas it is comparatively high, about 0.012 and 0.003 for fuzzy logic controllers and 0.3195 and 0.004 for conventional proportional integral controllers.

The stability analysis of fuzzy logic and genetic algorithms based non-linear controllers is discussed in this thesis work. Experiments on non-linear and optimal control of AC drive, speed control of turbine compressor system and speed control of DC servo motor have been implemented using evolutionary hybrid techniques and a comparative analysis is made with the conventional techniques. A comparative improvement has been noted in controlling the torque and flux errors of a non-linear AC drive using fuzzy logic strategy and hybrid fuzzy logic genetic algorithm techniques. Speed control of a typical turbine compressor system for controlling the outlet of gas is performed by using fuzzy logic strategy and hybrid fuzzy logic genetic algorithm techniques. When compared to conventional controllers, fuzzy logic provides better control on 
transient and steady state errors and the incorporation of genetic algorithms with fuzzy logic further optimizes the controller parameters. DC servo motor is optimized using fuzzy logic and hybrid fuzzy logic genetic algorithm techniques and compared with the conventional proportional integral controller. The novel fuzzy logic and hybrid fuzzy logic genetic algorithm techniques outperform the conventional approach in terms of minimization of transient and steady state errors.

\section{CONCluSion ANd Future Scope}

The stability analysis of fuzzy logic and genetic algorithms based non-linear controllers is discussed in this thesis work. Experiments on non-linear and optimal control of AC drive, speed control of turbine compressor system and speed control of DC servo motor have been successfully implemented using evolutionary hybrid techniques and a comparative analysis is made with the conventional techniques. A comparative improvement has been noted in controlling the torque and flux errors of a non-linear AC drive using fuzzy logic strategy and hybrid fuzzy logic genetic algorithm techniques. Speed control of a typical turbine compressor system for controlling the outlet of gas is performed by using fuzzy logic strategy and hybrid fuzzy logic genetic algorithm techniques. When compared to conventional controllers, fuzzy logic provides better control on transient and steady state errors and the incorporation of genetic algorithms with fuzzy logic further optimizes the controller parameters. DC servo motor is optimized using fuzzy logic and hybrid fuzzy logic genetic algorithm techniques and compared with the conventional proportional integral controller. The novel fuzzy logic and hybrid fuzzy logic genetic algorithm techniques outperform the conventional approach in terms of minimization of transient and steady state errors.

Abridged, it is evident from the results of the different case studies taken up in this work that the soft computing techniques are a great deal of research for tackling non-linear complex process control systems. A conclusion can be drawn that the fuzzy logic is most robust and systematic approach for controlling the process. In addition to fuzzy logic, hybridization with genetic algorithms, further provide better optimization of the errors.

\section{REFERENCES}

[1] Kumar K., Sakthibala D. and Palaniswami S., (2010), "Efficiency optimization of Induction Motor Drive using Soft computational techniques," International Journal of Computer Applications, 3(1), pp. 6-12.

[2] Arulmozhiyal R. and Baskaran K. , (2009), "Speed control of induction Motor using Fuzzy PI and Optimized using Genetic Algorithms," International Journal of Recent Trends in Engineering , 2(5), pp. 43-47.

[3] Pujar J.H and S.F. Kodad, (2009), "Digital Simulation of Direct Torque Fuzzy Control of PMSM Servo System” International Journal of Recent Trends in Engineering, 2(2), pp. 89-93.

[4] Balamurugan S., Xavier R. and Jeyakumar A., (2009), "Control of Heavy-duty Gas Turbine Plants for Parallel Operation Using Soft Computing Techniques", Electric Power Components and Systems, 37(11), pp. $1275-1287$.

[5] Esmaeili V., Assareh A., Shamsollahi M.B. Maoradi M.H. and Arefian N.M., (2008), "Estimating the depth of anesthesia using fuzzy soft computation applied to EEG features," Intelligent data Analysis 12, pp. 393-407. 
[6] Nagraj B., Subha S. and Rampriya B., (2008), “Tuning Algorithm for PID controller using soft computing Techniques," International Journal of Computer Science and network Security, 8(4), pp. 278-281.

[7] Yakhchali S. H. and Ghodsypour S. H.,(2008), "A Hybrid Genetic Algorithms for Computing the Float of an Activity in Networks with Imprecise Durations", Proceedings of the IEEE International Conference on Fuzzy Systems ,FUZZ-2008, pp.1789-1794.

[8] Yakhchali S. H. and Ghodsypour S. H., (2008), "A Hybrid Genetic Algorithms for Computing the Float of an Activity in Networks with Imprecise Durations", Proceedings of the IEEE International Conference on Fuzzy Systems, FUZZ-2008, pp.1789-1794.

[9] Rouabah Z., Zidani F. and Abdelhadi B., (2008), "Efficiency Optimization of Induction Motor Drive using Fuzzy Logic and Genetic Algorithms," IEEE International Symposium on Industrial Electronics, pp. 737-742.

[10] Toufouti R. Meziane S., (2006), "Direct torque control for Induction Motor using Fuzzy Logic," ACSE Journal, 6(2), pp. 19-26.

[11] Gang Feng, (2006), "A Survey on Analysis and Design of Model-Based Fuzzy Control Systems," IEEE Transactions on Fuzzy Systems, 14 (5 ), pp. 676 - 697.

[12] Vasudevan M., Argumugan R. and Paramasivam S. , (2005), "High- performance Adaptive Intelligent Direct torque schemes for Induction Motor Drives," Serbian Journal of Electrical Engineering , 2(1), pp. 93-116.

[13] Changliang X., Peijian G., Tingna S. and Mingchao W., (2004), "Speed control of brushless DC motor using genetic algorithm based fuzzy controller", Proceedings of the International Conference on Intelligent Mechatronics and Automation Chengdu,China, pp.460-464.

[14] Rekioua T. and Rekioua D., (2003), "Direct torque control strategy of permanent magnet synchronous machines," IEEE Bologna on Power Tech Conference Proceedings, 6(2), pp.6 -12.

[15] Behzad M., Mahdi J. and Farhad B., (2003) , “Application of Fuzzy Sliding Mode Based on Genetic Algorithms to Control of Robotic Manipulators", Proceedings of IEEE Conference on Emerging Technologies and Factory Automation, Vol.2, pp.169- 172.

[16] Yansheng Yang and Junsheng Ren, (2003), “Adaptive fuzzy robust tracking controller design via small gain approach and its application,” IEEE Transactions on Fuzzy Systems, 11 (6), pp.783 - 795.

[17] Gang Feng, (2003), "Controller synthesis of fuzzy dynamic systems based on piecewise Lyapunov functions and bilinear matrix inequalities," The 12th IEEE International Conference on Fuzzy Systems, 2003, FUZZ-03, vol.2, pp.1327 - 1332.

[18] Chang Wook Ahn and Ramakrishna, R.S., (2002), "A genetic algorithm for shortest path routing problem and the sizing of populations," IEEE Transactions on Evolutionary Computation, 6(6), pp. 566-579.

[19] Chia-Feng Juang, (2002), “A TSK-type recurrent fuzzy network for dynamic systems processing by neural network and genetic algorithms," IEEE Transactions on Fuzzy Systems, 10 (2), pp. 155-170.

[20] Ganguli R., (2002), "Fuzzy Logic Intelligent System for Gas Turbine Module and System Fault Isolation", Journal of Propulsion and Power, 18(2), pp. 1-6.

[21] Kewley R. and Embrechts J. and Kewley, R.H., Embrechts, M.J., (2002), "Computational military tactical planning system," IEEE Transactions on Systems, Man, and Cybernetics, Part C: Applications and Reviews, 32( 2), pp.161- 171.

[22] Adams J. M. and Rattan K. S., (2001), "Genetic multi-stage Fuzzy PID controller with a Fuzzy switch”, IEEE Transactions on Systems, Man, and Cybernetics, Vol. 4, pp.2239-2244.

[23] Liu B., Chen C. and Tsao J., (2001), "Design of Adaptive Fuzzy Logic Controller Based on Linguistic-Hedge Concepts and Genetic Algorithms," IEEE Transactions On Systems, Man, And Cybernetics-Part B: Cybernetics, 31(1),pp. 32-53. 
[24] Rubaai A., Ricketts D. and Kankam M.D., (2001), "Experimental verification of a hybrid fuzzy control strategy for a high-performance brushless DC drive system," IEEE transactions on Industry Applications," 37(2), pp. 503-512.

[25] Zhanli J., Yaowen Y. and Chee K., (2000), “Application of Fuzzy GA for optimal vibration control of smart cylindrical Shells", School of Civil and Environmental Engineering, Nanyang Technological University, Singapore, 14(6), pp. 1250.

[26] El-Shal S.M., Morris and A.S., (2000), "A fuzzy expert system for fault detection in statistical process control of industrial processes," IEEE Transactions on Systems, Man, and Cybernetics, 30(2), 1094-1097.

[27] Oh W., Kim Y., Kim C., Kwon T. and Kim H., (1999), "Speed control of induction motor using Genetic Algorithms based Fuzzy controller," Proceedings of the 25th Annual Conference of the IEEE, Industrial Electronics Society, Vol.2, pp.625 - 629.

[28] Jung-Hsian Chiang, (1999), "Choquet fuzzy integral-based hierarchical networks for decision analysis," IEEE Transactions on Fuzzy Systems, 7(1), pp.63- 71.

[29] Barolli L., Koyama A., Motegi S. and Yokoyama S., (1998), "An intelligent policing-routing mechanism based on Fuzzy Logic and Genetic Algorithms," Proceedings of the International Conference on Parallel and Distributed Systems, pp.390-397.

[30] Guo J. and Huang S.H., (1996), "Control of an Autonomous Underwater Vehicle Testbed Using Fuzzy Logic and Genetic Algorithms," Proceedings of the Symposium on Autonomous Underwater Vehicle Technology, pp. 485 - 489.

[31] Freisleben B. and Strelen S., (1995), “A Hybrid Genetic Algorithms/Fuzzy Logic Approach to Manufacturing Process Control," IEEE International Conference on Evolutionary Computation, Vol.2, pp.837-841.

[32] Hwang W. and Thompson W. E., (1994), "Design of Intelligent Fuzzy Logic Controllers Using Genetic Algorithms," Proceedings of the Third IEEE Conference at IEEE World Congress on Computational Intelligence and Fuzzy Systems, 1994, vol.2, pp. 1383-1388.

[33] Merrington, G. L., (1993), "Fault Diagnosis in Gas Turbines Using a Model Based Technique," American Society of Mechanical Engineers, ASME Paper.

[34] Habetler T.G., Profumo F., Pastorelli M., and Tolbert L.M., (1992), "Direct Torque Control of Induction Machines using Space Vector Modulation," IEEE Transactions on Industry Applications, 28(5), pp. $1045-1053$. 


\section{Authors}

Rahul Malhotra is currently working as Assistant Professor \& Head, Bhai Maha Singh College of Engineering, Muktsar. His area of interest includes Wireless Communication, Adhoc Networks, and Fuzzy Logic \& Genetic Algorithms. He is a member of Indian Society for Technical Education (ISTE), The Institution of Electronics \& Telecommunication Engineers (India), Institution of Engineers (India).

E-mail: blessurahul@gmail.com

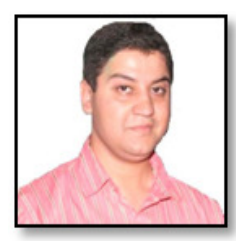

Yaduvir Singh is currently working as Associate Professor in Thapar University, Patiala, Punjab. He as a teaching faculty served Institute of Engineering \& Technology, Lucknow, NE Regional Institute of Science and Technology, Itanagar, GB Pant Engineering College, Pauri Garhwal, Harcourt Butler Technological Institute, Kanpur before joining Thapar University as Assistant Professor in 2000. His areas of interest includes neural networks, fuzzy logic optimization, power systems, automated control systems, industrial electronics etc.

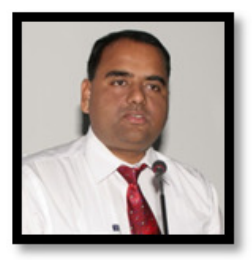

E-mail: yad_pra@yahoo.com

Narinder Singh is currently working as Director at GGS group of Colleges, Talwandi Sabo. In 1964 he joined as a Lecturer and in 1982 he joined as Assistant Professor in Thapar Institute of Technology, Patiala. He joined as Professor in Thapar in 1991 and shifted to Giani Zail Singh College of Engineering \& Technology, Bathinda as Professor in 1993. He served there as Professor and Head (1994-1998) and as Principal (1998-2002). Later, he joined as Principal in GGS College of Engineering \& Technology, Talwandi Sabo, Bathinda in 2002.

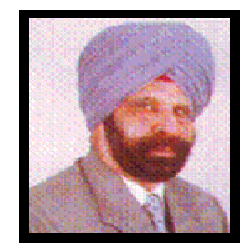

$\mathrm{He}$ is a fellow member of Institution of Engineers (India), Indian Society for Technical Education, Institute of Surveyors and Chairman (Board of studies), Punjabi University, Patiala, Sant Longowal Institute of Engineering \& Technology, Sangrur. His field of interest includes Surveying, Fuzzy Logic, Genetic Algorithm optimization. 